\title{
AUTONOMÍA PERSONAL DE LAS MUJERES CON DISCAPACIDAD: EL CASO DE LA ACCESIBILIDAD A LAS AYUDAS TÉCNICAS Y NUEVAS TECNOLOGÍAS
}

\author{
ELVIRA RAMOS GARCÍA \\ Universidad de Murcia
}

Recibido: 16/03/2009

Aceptado: 28/05/2009

\section{Introducción}

El Manifiesto de las Mujeres con Discapacidad en Europa ${ }^{1}$, en el apartado de «La autonomía, la asistencia personal, las necesidades de asistencia técnica y asesoramiento», desarrolla los objetivos que deben alcanzarse para conseguir la autonomía personal de las personas con discapacidad ${ }^{2}$.

En el presente trabajo pretendemos analizar la situación de accesibilidad a las ayudas técnicas y nuevas tecnologías ${ }^{3}$, así como el asesoramiento para

1. Foro Europeo de la Discapacidad, «El Manifiesto de las Mujeres con Discapacidad en Europa». Bruselas, 1998.

2. Teniendo en cuenta que las mujeres con discapacidad tienen menor acceso a equipos y ayudas técnicas, los equipos necesarios deben ser producidos localmente y facilitados a todas las mujeres con discapacidad a un precio lo suficientemente bajo como para que éstas puedan adquirirlos, o de manera gratuita. Se hace hincapié en la puesta en marcha de programas regionales y nacionales de fabricación de equipos y ayudas técnicas, que también abarquen la formación de las mujeres con discapacidad para la fabricación y utilización de dicho material. Las ayudas técnicas deben satisfacer las necesidades funcionales de las mujeres y obedecer a criterios estéticos en lo que a su diseño, material y calidad se refiere. Si estas ayudas técnicas no estuvieran disponibles localmente, se debe facilitar el suministro de equipos importados allá donde sean necesarios, en el trabajo o en el hogar.

3. No hacemos distinción entre ayudas técnicas y nuevas tecnologías porque la mayoría de los caso las ayudas técnicas tienen incorporadas nuevas tecnologías. Así evitamos que el término de «nuevas tecnologías» esté aplicado exclusivamente al como de la Información y Comunicación.

Feminismo/s 13, junio 2009, pp. 171-186 
la autonomía personal de las mujeres con discapacidad, con el fin de realizar propuestas y de agilizar la solución de los déficits que en esta materia se están produciendo.

Teniendo en cuenta que en el ámbito social las mujeres con discapacidad soportan una doble discriminación por género y discapacidad, señalada en múltiples trabajos ${ }^{4}$, y ello sin entrar en la múltiple discriminación referida a etnia, orientación sexual, edad, lugar de residencia, situación laboral, nivel socioeconómico y otras.

No estamos en condiciones de abordar la accesibilidad a las ayudas técnicas según la múltiple discriminación, pues está siendo objeto de un estudio en marcha. Sin embargo, podemos referirnos a una cuestión empírica, el hecho de que las ayudas técnicas son un artículo de lujo, por tanto, solo accesibles a los sectores de alto nivel socioeconómico. La mujer, al encontrarse en el sector socioeconómico más bajo ${ }^{5}$, si además tiene una discapacidad entra en el terreno de la exclusión social ${ }^{6}$.

Por otra parte, no podemos dejar de señalar que la globalización está siendo un elemento de incremento de desigualdades de los colectivos más vulnerables, como el de las mujeres con discapacidad, tal y como está evidenciando el índice de desarrollo humano (IDH) y el índice de desarrollo humano relativo a género (IDG) ${ }^{7}$.

Hace once años de la declaración del Manifiesto de la Mujeres con Discapacidad en Europa, seis años desde que apareciera el trabajo «Mujer y Discapacidad, Buenas prácticas ${ }^{8}$ del Observatorio de la Discapacidad y cuatro años desde la presentación del trabajo «Ayudas Técnicas y discapacidad $»^{9}$ del CERMI. Sin embargo, vemos cómo el progreso en la solución de los graves problemas que presenta la accesibilidad de las ayudas técnicas a las muje-

4. Como se señaló en el Manifiesto citado en la nota 1 y como señala Marita Iglesias en su trabajo La importancia de ser mujer. Una reflexión desde la diversidad funcional. Presentado en el Foro Internacional sobre comunicación e discapacidades, celebrado el 1 de noviembre de 2006 en Pontevedra.

5. Como refieren los trabajos de la pobreza de las mujeres, entre los que destacamos: ElEJABEITIA, C. Feminización de la pobreza. Documentación Social, 195(1996), pp 171-182. Y GARCíA-MinA, A. (Coord.) Género y desigualdad: la feminización de la pobreza. Madrid, Universidad Pontificia de Comillas, 2004.

6. Es interesante en este sentido el libro de Jordi Estivill, Panorama de la lucha contra la exclusión social. Conceptos y estrategias. Organización Internacional del Trabajo, 2003.

7. Los importantes índices de desarrollo humano elaborados por el PNUD.

8. IMSERSO. «Mujer y Discapacidad. Buenas Prácticas». Madrid, Ministerio de Trabajo y Asuntos Sociales, 2000.

9. Pérez Bueno, L. C. Ayudas Técnicas y Discapacidad. Madrid, CERMI, 2005. 
res con discapacidad avanza lentamente ${ }^{10}$. A las drásticas medidas que las Administraciones Públicas debieran acometer para solucionar la accesibilidad a la ayudas técnicas se une la ancestral discriminación de las mujeres propia de la subordinación patriarcal. Vencer la doble discriminación de mujer y discapacidad en el terreno de la accesibilidad a las ayudas técnicas ha de ser nuestro principal objetivo.

\section{Justificación del trabajo: marco legal}

Para lograr la autonomía e independencia de la mujer con discapacidad, mediante el uso de las ayudas técnicas, tenemos como fundamento de nuestro trabajo la legislación vigente ${ }^{11}$ internacional, europea y nacional dictada hasta este momento.

En el ámbito Internacional nos regimos por la Convención de Naciones Unidas sobre Derechos de las Personas con Discapacidad ${ }^{12}$ en 2006 y el Congreso Internacional de Personas con Discapacidad en Madrid de 2003 ${ }^{13}$. Del mismo modo, también encontramos las Normas Uniformes sobre la igualdad de oportunidades para las personas con discapacidad ${ }^{14}$, la Resolución del Consejo de 6 de febrero de 2003 sobre «Accesibilidad electrónica» ${ }^{15}$ y la Convención Internacional sobre los Derechos de las Personas con Discapacidad de $2006^{16}$.

10. Aunque, sorprendentemente, el avance es también muy lento para los hombres con discapacidad, dado que el sector de las ayudas técnicas adolece de racionales estructuras. Es más las irracionalidades estructurales que existen en el sector exigen reformas drásticas por parte de las Administraciones Públicas.

11. Para más información sobre legislación vigente de aspectos parciales consultar la Web. <http://usuarios.discapnet.es/disweb2000/index.htm>, consultada el 30-04-2009.

12. Convención de las personas con discapacidad. Asamblea General de la ONU el 13 de diciembre de 2006.

13. Congreso Internacional de Personas con Discapacidad. «No discriminación más acción positiva es igual a inclusión social». Madrid, 2003.

14. Las Normas Uniformes sobre la igualdad de oportunidades para las personas con discapacidad fueron aprobadas por la Asamblea General de las Naciones Unidas en su cuadragésimo octavo período de sesiones, mediante resolución 48/96, del 20 de diciembre de 1993 (publicada en el documento A/RES/48/96, de 4/03/1994).

15. El 13 de diciembre de 2006, la Asamblea General de la ONU adoptó la Convención Internacional sobre los Derechos de las Personas con Discapacidad, un avance histórico para más de 650 millones de mujeres y hombres con discapacidad.

16. I Plan Nacional de Accesibilidad 2004-2012, aprobado en Consejo de Ministros el día 25 de julio de 2003. 
Respecto a las normas de ámbito nacional, encontramos el I Plan Nacional de Accesibilidad 2004-2012 $2^{17}$, IV Plan Nacional de Acción para la Inclusión Social del Reino de España (2006-2008), Plan de Acción para las Mujeres con discapacidad 2007 aprobado por el Consejo de Ministros de 1 de diciembre de 2006, el RD. 1865/2004 por el que se regula el Consejo Nacional de la Discapacidad ${ }^{18}$ y las leyes de Medidas de Protección Integral contra la Violencia de Género ${ }^{19}$, de Promoción de la Autonomía Personal y Atención a Personas en Situación de Dependencia ${ }^{20}$ y la Ley para la igualdad efectiva de mujeres y hombres ${ }^{21}$. Esa misma ley proclama como principio inspirador de las políticas públicas sobre discapacidad el diálogo civil y ordena la participación activa de las personas con discapacidad, a través de sus organizaciones representativas, en todos los asuntos y materias que les afecten directa o indirectamente. Además, también incorporamos lo regulado acerca de los Productos Sanitarios ${ }^{22}$.

\section{Resultados}

\subsection{Diagnóstico de las mujeres con discapacidad y la utilización de ayudas técnicas}

El informe previo del Instituto Nacional de Estadística de 2008, acerca de la utilización de las ayudas técnicas por parte las personas con discapacidad, describe tanto la magnitud de la discapacidad, como la accesibilidad a las ayudas técnicas. En la tabla 1 acerca de las discapacidades en España de las personas mayores de 6 años de edad se constata que de los 3.787 .400 millones

17. Real Decreto 1865/2004, de 6 de septiembre, por el que se regula el Consejo Nacional de la Discapacidad.

18. Ley Orgánica 1/2004, de 28 de diciembre, de Medidas de Protección Integral contra la Violencia de Género.

19. Ley 39/2006 de Promoción de la Autonomía Personal y Atención a personas en situación de dependencia, publicada en el BOE número 299 de 15 de diciembre de 2006.

20. Ley Orgánica 3/2007, de 22 de marzo, para la igualdad efectiva de mujeres y hombres. En el artículo 8 de la Ley de igualdad de oportunidades, refiere que las ayudas técnicas son una clase de medida de «acción positiva» consistente «en apoyos de carácter específico destinados a prevenir o compensar las desventajas o especiales dificultades que tienen las personas con discapacidad en la incorporación y participación plena en los ámbitos de la vida política, económica, cultural y social».

21. El Real Decreto 414/1996 da una definición que afecta a todas las ayudas técnicas, partiendo del beneficio generado por éstas para el usuario y sin diferenciar entre clases de ayudas técnicas. El Real Decreto 2727/1998 introduce algunas modificaciones sobre el anterior. Una nueva modificación aparece en el RD 1662/2000, de 29 de Septiembre, sobre productos sanitarios para diagnóstico in «vitro».

22. INE: INEBASE: Avance de resultados de la Encuesta de Discapacidad, Autonomía personal y situaciones de Dependencia (EDAD) 2008. Consulta a 5 de noviembre de 2008. 
de personas con discapacidad, las mujeres son 2.276 .500 (53.4\%). En relación con la accesibilidad a las ayudas técnicas (tabla 2 y gráficol), según los datos del INE de 2008, observamos que las mujeres sólo reciben el 10.3\% de las ayudas técnicas, dato que supone un $24 \%$ menos que los hombres.

No obstante, es llamativo el hecho de que sólo alrededor del 10\% de las personas con discapacidad accedan a herramientas tan importantes para su autonomía personal; se evidencia lo que esperábamos, la distinta accesibilidad a las ayudas técnicas por sexo.

Tabla 1 : Personas con discapacidad de 6 y más años por sexo

\begin{tabular}{|c|c|c|}
\hline \multicolumn{3}{|c|}{ Tabla 1. Personas con discapacidad de 6 y más años por sexo } \\
\hline & Números absolutos & $\%$ \\
\hline Total & 3.787 .400 & 100 \\
\hline Mujeres & 2.276 .500 & 53,39 \\
\hline Hombres & 1.510 .900 & 46,31 \\
\hline
\end{tabular}

Fuente: INE: INEBASE: Avance de resultados de la Encuesta de Discapacidad, Autonomía personal y situaciones de Dependencia (EDAD) 2008. Consulta a 5 de noviembre de 2008. Elaboración Propia.

Gráfico 1. Personas con discapacidad que utilizan ayudas técnicas por sexo

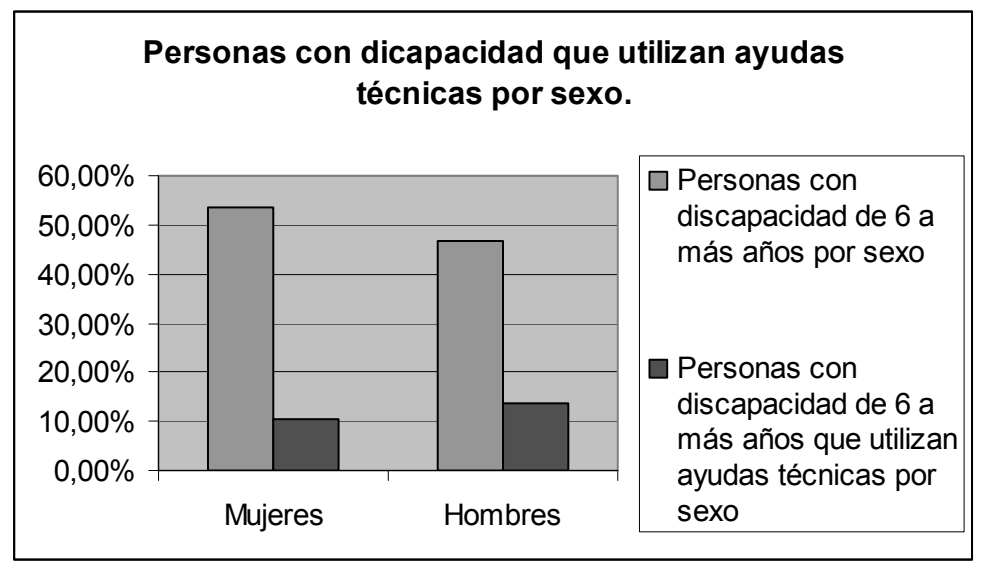

Fuente: INE: INEBASE: Avance de resultados de la Encuesta de Discapacidad, Autonomía Personal y Situaciones de Dependencia (EDAD) 2008. Consulta, el 5 de noviembre de 2008. Elaboración Propia. 


\subsection{Principales problemas de la accesibilidad a ayudas técnicas}

A partir de la legislación anteriormente citada, las ayudas técnicas han pasado a ser herramientas fundamentales para el logro de la vida independiente y la integración social de las mujeres con discapacidad. En este nuevo escenario, cabe decir que el acceso, uso y disfrute de las mismas, la mayoría de ellas con nuevas tecnologías incorporadas, deben constituir un derecho subjetivo y garantizado por ley para las mujeres con discapacidad. Este derecho ha de ser universal y gratuito, además de exigible y vinculable a las Administraciones Públicas. Tal y como recoge el artículo 8 de la Ley 51/2003, de 2 de diciembre, «de igualdad de oportunidades, no discriminación y accesibilidad universal de las personas con discapacidad».

Vamos a centrarnos en las principales conclusiones del trabajo de «Ayudas Técnicas y discapacidad» del CERMI ${ }^{23}$, en el que se lleva a cabo un análisis exhaustivo del estado del mercado de las ayudas técnicas. Las principales características del sector de las ayudas técnicas desde el punto de vista de las mujeres con discapacidad son:

\subsubsection{Falta de información de las personas con discapacidad}

Existe una escasa información entre las mujeres con discapacidad sobre la propia existencia de las ayudas técnicas y de otras prestaciones similares, puntos de venta, ayudas económicas, etc.

\subsubsection{Falta de información de los profesionales}

También ignoran estos recursos la mayoría de los profesionales que deberían realizar una labor informativa en esta materia: personal de trabajo social, de medicina, etc.

\subsubsection{Escasa información sectorial}

Las asociaciones empresariales del sector y las instituciones públicas responsables de la regulación y prestación de este tipo de servicios, no divulgan de manera adecuada la información que permita conocer, diversos datos sobre las mismas, como su volumen de negocio o el gasto de la Administración Pública por Comunidad Autónoma y tipo de ayuda ${ }^{24}$.

23. Op. cit.

24. Baste observar el listado de ayudas técnicas que proporciona el CEAPAT (Centro Estatal de Autonomía Personal y Ayudas Técnicas) en su página Web <http://www.catalogo- 
Existen algunas excepciones ${ }^{25}$ en empresas del sector, que avanzan hacia la consecución de una estructuración del mercado similar al de otros países europeos, reduciendo sus costes facilitando así su accesibilidad.

\subsubsection{Precio excesivo de las ayudas técnicas}

Las ayudas técnicas tienen unos elevados márgenes comerciales, muy variables según empresas, lo que las convierte en artículos de «lujo» inalcanzables mediante la financiación privada para la mayor parte de las personas con discapacidad, sobre todo para las mujeres.

\subsubsection{Escasa financiación pública para la adquisición de ayudas técnicas}

En relación con la financiación pública para la adquisición de ayudas técnicas, tomamos como ejemplo los Catálogos de Prestaciones Ortoprotésicas en las diferentes Comunidades Autónomas. Sus criterios de confección son económicos y atienden a un equilibrio presupuestario, sin tener en cuenta, ni valorar el bienestar y las oportunidades de integración social que estas ayudas pueden aportar a las personas que las necesitan. Existen grandes diferencias en el coste financiero de estas ayudas técnicas, lo que da lugar a desigualdades territoriales entre las distintas comunidades autónomas.

\subsubsection{Inexistencia de canales especializados de venta}

La escasa competencia existente entre los operadores del sector genera una falta de atención en criterios de calidad y rapidez en el servicio. Otra dificultad es el elevado número de referencias de ayudas técnicas existentes, la gran variedad de discapacidades que éstas han de cubrir y la especialización técnica que están requiriendo los avances tecnológicos. Por todo ello es necesaria la aparición de operadores especializados por tipo de ayuda técnica o discapacidad, para facilitar el suministro y capacitación en la fabricación y adaptación de las ayudas.

ceapat.org/> consultado el 30-04-2009, experimentar la complejidad de catalogación, y por ende de información y accesibilidad.

25. No entramos en este momento a señalar su personalidad jurídica. 
3.2.7. Ausencia de ventajas o servicios adicionales a la venta de la ayuda técnica

La falta de competitividad señalada anteriormente conlleva un desarrollo muy limitado de servicios accesorios a la venta de las ayudas técnicas que repercuta en un mejor servicio de post-venta.

3.2.8. Repercusión de los problemas de las mujeres con discapacidad en el conjunto de la sociedad

Los problemas enumerados, hasta el momento, están obligando a los compradores de ayudas técnicas (públicos o privados) a asumir costes e ineficiencias inaceptables en una economía de libre mercado.

Por otra parte, el alto coste de estas ayudas reduce la financiación de las mismas tanto por entidades privadas como públicas, lo que se traduce directamente en un incremento significativo de cuotas y limita su accesibilidad por parte, en este caso, de las mujeres. Respecto a las Comunidades Autónomas, es decir respecto a la financiación pública, los problemas van a ser similares, puesto que el alto coste de estas ayudas reduce la capacidad de compra de los servicios de salud y aumenta los costes del tratamiento de la discapacidad. Todo ello constituye un impedimento objetivo al desarrollo de la autonomía personal de las mujeres con discapacidad, impedimento que trasciende el ámbito personal afectando a sus familias también.

\section{Estrategias de mejora}

Siguiendo el trabajo realizado por $\mathrm{CERMI}^{26}$, planteamos la necesidad de realizar una serie de propuestas encaminadas a mejorar sustancialmente esta situación de inaccesibilidad a las ayudas técnicas por parte de las mujeres con discapacidad. Nuestra aportación a estas propuestas del CERMI consiste en integrar en ellas la perspectiva de género A partir de lo analizado en los foros feministas se ha puesto en evidencia que las mujeres sufren una doble discriminación: como mujeres y como personas con discapacidad. Proponemos que las mujeres con discapacidad sean las auténticas protagonistas de su proceso de empoderamiento por el cual alcancen el mayor grado posible de autonomía personal. De esta manera las mujeres con discapacidad estarán en el epicentro de las políticas de accesibilidad a los bienes y servicios que necesiten, lo que servirá también para deconstruir las desigualdades de género en esta materia.

Las propuestas que presentamos se pueden resumir en:

26. Op. cit.

Feminismo/s 13, junio 2009, pp. 171-186 


\subsection{Participación de las mujeres con discapacidad en la planificación de las acciones de mejora de accesibilidad a las ayudas técnicas}

La primera cuestión que debe resolverse es la de caminar hacia la igualdad de mujeres y hombres con discapacidad, venciendo todos los obstáculos de discriminación mencionados al principio del trabajo ${ }^{27}$.

En este sentido, la propuesta más importante de nuestro trabajo es la de la participación ${ }^{28}$ de las mujeres con discapacidad en la planificación de las acciones de mejora de accesibilidad a las ayudas técnicas. Y ello porque hemos constatado la nula presencia y participación de las personas con discapacidad, en particular y en general, otros interesados como profesionales, sectores empresariales etc. en la planificación, ejecución y evaluación de los procesos de acceso a los recursos tecnológicos ${ }^{29}$. La participación de las mujeres es fundamental, sólo contando con ellas, con sus organizaciones y con sus familias nos podremos asegurar que la gestión de los recursos es eficaz. Al empoderamiento $^{30}$ de las mujeres con discapacidad se ha de unir el esfuerzo organizado de sus organizaciones y sus familias, teniendo una activa participación en la gestión de estas ayudas, asumiendo responsabilidades y siendo copartícipes del proceso. Esta posición viene avalada legalmente por la Ley 51/2003, de 2 de diciembre, de igualdad de oportunidades, no discriminación y accesibilidad universal de las personas con discapacidad.

\subsection{Creación de un Centro Estatal Público de Referencia en materia de ayudas técnicas}

Proponemos la creación de un Centro de Referencia en materia de ayudas técnicas de la Administración General del Estado, que debería asumir el propio CEAPAT modificando sus funciones, de manera que fuese posible la realización de la formación, desarrollo de la investigación, información, difusión y elaboración de normas técnicas, fomento de la calidad y de la satisfacción del

27. En este sentido vamos más lejos que la legislación española que sólo da esa participación a través de las organizaciones de las mujeres, nosotros pensamos que a veces esta fórmula es insuficiente para una verdadera democracia participativa.

28. LATHER,P. «Research as Praxis». Harvard Educational Review, 56 (1986), pp. 257-277; Ramos García, E., SÁnchez Moreno A., Marset Campos P. «Ciudad, salud y participación». Ciudad y territorio, 89 (1991), pp. 157- 167.

29. El empoderamiento de las mujeres con discapacidad en cualquier proceso que les afecte es la herramienta fundamental para el éxito.

30. RAMOS GARCía E., SÁNCHEZ MORENO, A. «Investigación acción, participación (IAP) en la promoción de salud de las mujeres». En I Congreso Nacional La salud de las mujeres: hacia la igualdad de género en salud. Instituto de la Mujer, Madrid, 2002. 
usuario, evaluación, prospectiva, cooperación con otras administraciones y con la sociedad civil, etc.

\subsubsection{Unificación del concepto de ayuda técnica}

Insistimos, asimismo, en la necesidad de definir y unificar el concepto de ayuda técnica ${ }^{31}$, para que las acciones que se realicen, con objeto de facilitar el acceso de las mujeres con discapacidad a este tipo de bienes y servicios, se extiendan y repercutan en su beneficio, independientemente de la discapacidad y del tipo de ayuda técnica requerido.

La justificación a esta propuesta está basada en la definición que realiza de los Productos Sanitarios el Real Decreto 414/1996, que afecta a todas las ayudas técnicas, partiendo del beneficio generado al usuario, en nuestro caso usuaria.

\subsubsection{Elaboración de un Catálogo General socio-sanitario de ayudas técnicas}

Consideramos que la elaboración de un Catálogo General socio-sanitario de ayudas técnicas ${ }^{32}$, aparte de recoger los diferentes tipos de ayudas técnicas existentes, debería incluir los tipos de deficiencias y discapacidades ${ }^{33}$ para las que es aplicable cada una de las ayudas técnicas, el grado de mejora de la discapacidad generado por cada una de las ayudas técnicas y componentes sociales y personales (actividad laboral, aficiones, perfil personal, etc.) relativos a la utilización de dichas ayudas técnicas ${ }^{34}$.

De esta forma, las mujeres con discapacidad dispondrían de una referencia en cuanto a los tipos de ayudas técnicas existentes y la funcionalidad ofrecida por cada una de ellas. Así podrían disponer de la información suficiente

31. Como comentamos anteriormente en la complejidad del CEAPAT.

32. La elaboración de un Catálogo General Socio-sanitario de ayudas técnicas, debería ser promovido desde la Administración General del Estado, por el Ministerio de Sanidad y Política Social, y en cuya elaboración deben participar todos los operadores del sector: Administraciones Autonómicas, fabricantes, comercializadores, usuarios, clientes, prescriptores, etc. Sería el Catálogo de Referencia del Sistema Nacional de Salud, del Sistema Nacional de Servicios Sociales (por crear) y el Sistema Nacional de Atención a las Situaciones de Dependencia (por crear).

33. Para el detalle de la clasificación de las discapacidades visitar la página Web: <http://sid. usal.es/colectivos.aspx > consultado el 30-04-2009.

34. El objeto de este Catálogo sería el identificar la utilidad de las ayudas técnicas y evaluar en qué grado cumple cada una de ellas con los objetivos propuestos por las Naciones Unidas en cuanto a la inclusión y la vida independiente y autónoma de las personas con discapacidad. Igualmente sería útil para otros actores de este sector. 
como para valorar y decidir cuál es la ayuda técnica más adecuada de acuerdo a sus necesidades particulares.

Con este catálogo, que indudablemente supondrá una inversión de tiempo y de dinero, mejorará la accesibilidad y autonomía de las mujeres con discapacidad.

\subsubsection{Catálogo de Prestaciones Ortoprotésicas de los Servicios públicos de} las Comunidades Autónomas como mecanismo regulador de precios

El Catálogo de Prestaciones Ortoprotésicas de las Comunidades Autónomas tiene un enorme potencial como herramienta correctora de los desajustes del mercado de las ayudas técnicas y como arma de la Administración Autonómica para la defensa de los intereses de las mujeres con discapacidad ${ }^{35}$.

Aun cuando la competencia de esta materia esté en las Comunidades Autónomas, éstas deberán elaborar un protocolo común con el fin de evitar desigualdades territoriales en la accesibilidad a estas ayudas por parte las mujeres con discapacidad. Nuestra propuesta va encaminada a que las ayudas técnicas las financie la administración pública. Ello exige modificaciones en los criterios de adjudicación y en el personal técnico que las valore. Las Comunidades Autónomas deberán señalar tales criterios, así como los profesionales adecuados.

\subsubsection{Formación sobre las ayudas técnicas para mejorar su accesibilidad}

Mientras la participación de las mujeres con discapacidad descrita en la primera propuesta se ponga en marcha, de forma transitoria habría que establecer un programa de formación para que todos los profesionales implicados tuvieran las habilidades necesarias en la comunicación de la información, encaminado a que las mujeres con discapacidad dispongan de información suficiente y adaptada de forma comprensible para todas las destinatarias a la hora de acceder a una ayuda técnica, independientemente de quién la financie. El objetivo ha de ser que las mujeres con discapacidad puedan participar en la decisión de elección de la ayuda técnica, para que ésta se pueda adaptar a sus necesidades atendiendo a sus expectativas.

35. A la hora de incluir una Ayuda Técnica en el Catálogo de Prestaciones Ortoprotésicas y valorar la prestación económica de ésta, se realizará un análisis económico comparativo con otras prestaciones, con el fin de ajustar los precios de este sector y abaratar costes con todos los beneficios que ello comporta de cara a las usuarias de ayudas técnicas, etc. 
Los profesionales objeto de formación han de ser los que permitan que las usuarias de la ayuda técnica tengan el acceso a la información con plenas garantías de imparcialidad en el proceso de elección de ésta ${ }^{36}$. Es muy importante que la toma de decisión radique en las mujeres con discapacidad sin tutorías externas.

\subsection{Cooperación entre los sectores público y privado: racionalización de las ayudas técnicas}

A tenor de lo analizado anteriormente sobre los proveedores de ayudas técnicas, la escasa competencia que existe entre las empresas del sector, el alto coste de las ayudas técnicas, la falta de especialización, la falta de oferta de productos, y la carencia de servicios accesorios de venta de la ayuda técnica, se precisa la racionalización del sector, siendo deseable el carácter eminentemente público el mismo, idea por la que abogan y con la que cooperan las asociaciones de mujeres con discapacidad. Ello abarataría costes, y por lo tanto favorecería el incremento de la accesibilidad de las mujeres.

\subsection{Mejora del régimen fiscal aplicable a las ayudas técnicas}

Mientras la adquisición de las ayudas técnicas sea financiada privadamente por las mujeres con discapacidad habrían de promoverse reducciones fiscales. Para ello es preciso ampliar la relación de bienes, elementos y artículos considerados como ayudas técnicas, ahora excesivamente limitada, así como extender la aplicación de tipos muy reducidos en impuestos con clara incidencia en el coste del producto, como es el caso del IVA. Esta medida tendrá, por todo lo analizado anteriormente de la situación de pobreza de las mujeres con discapacidad, un efecto poco importante sobre ellas, pues la mayoría no tienen obligación de declarar a Hacienda.

36. Los profesionales de la medicina, el personal de trabajo social y de terapia ocupacional deberán tener un mayor conocimiento de las ayudas técnicas existentes y de los avances tecnológicos que se van produciendo, así como de las modalidades de realización, fabricación y adaptación de las ayudas técnicas de un mismo tipo, para que puedan, tras la prescripción, realizar un seguimiento de la misma y determinar si cumple todas las especificaciones precisas en cuanto a utilidad, calidad, tecnología aplicada en la fabricación y ergonomía, y evaluar su garantía. La labor de información a las mujeres con discapacidad deberá ser completada por las asociaciones de personas con discapacidad y por sus familias. 


\section{Conclusiones}

La equiparación social plena de las mujeres con discapacidad se alcanzará mediante la eliminación de los obstáculos en los ámbitos de la vida social, cultural etc. Una de las herramientas fundamentales para lograrla son las ayudas técnicas, por lo que el desarrollo de medidas que contribuyan a su planificación y ordenación redundará en la consecución de dicha equiparación, tendrá un efecto multiplicador.

La accesibilidad igualitaria entre mujeres y hombres a este proceso de autonomía personal es una garantía, no la única, de que hemos actuado acertadamente en la no discriminación de las mujeres con discapacidad.

\section{Agradecimientos}

Agradezco a Josefa Belmonte Ortiz, diplomada en Maestra especialista de Educación Espacial por sus interesantes observaciones y asesoramiento en el presente trabajo.

\section{Referencias bibliográficas}

Congreso Internacional sobre Mujer y Discapacidad. Conclusiones del «I Congreso Internacional sobre Mujer y Discapacidad»: Generalitat Valenciana, Conselleria de Bienestar Social, Valencia, 27 de febrero a 1 de marzo de 2003. Alternativas. Cuadernos de Trabajo Social, 11 (dic. 2003).

Elejabeitia, C. «Feminización de la pobreza». Documentación Social, 105 (1996), pp. 171-182.

EstiviLL, J. Panorama de la lucha contra la exclusión social. Conceptos y estrategias. Organización Internacional del Trabajo, 2003.

García-Mina, A. (Coord.) Género y desigualdad: la feminización de la pobreza. Madrid, Universidad Pontificia de Comillas, 2004.

Foro EUROPEO DE LA DisCAPACIDAD. El Manifiesto de las Mujeres con Discapacidad en Europa. Bruselas, 1998.

IGLESIAS PADRON, M. «La importancia de ser mujer. Una reflexión sobre la discapacidad funcional»- Actas Foro Internacional sobre comunicación e discapacidades. Pontevedra, 2007, pp. 221-230.

IMSERSO. MUJER y DisCAPACIDAD. BUENAS PRÁCtICAS. MAdRID, Ministerio de Trabajo y Asuntos Sociales, 2000.

INE: INEBASE: Avance de resultados de la Encuesta de Discapacidad, Autonomía personal y situaciones de Dependencia (EDAD). 2008. Consulta a 5 de noviembre de 2008.

LAther, P. «Research as Praxis». Harvard Educational Review, 56 (1986) pp. 257-277.

Feminismo/s 13, junio 2009, pp. 171-186 
Pérez Bueno, L. C. Ayudas Técnicas y Discapacidad. Madrid, CERMI, 2005.

Ramos García E., SÁNCHEz Moreno, A. «Investigación acción, participación

(IAP) en la promoción de salud de las mujeres». En I Congreso Nacional «La salud de las mujeres: hacia la igualdad de género en salud». Madrid, Instituto de la Mujer, 2002.

Ramos García E., SÁnchez Moreno, A., Marset Campos, P. «Ciudad, salud y participación». Ciudad y territorio, 89 (1991), pp. 157- 167.

\section{Legislación}

Normas Uniformes sobre la igualdad de oportunidades para las personas con discapacidad fueron aprobadas por la Asamblea General de las Naciones Unidas. 20 de diciembre de 1993 (publicada en el documento A/RES/48/96, de 4/03/1994).

Resolución del Consejo de 6 de febrero de 2003 sobre «Accesibilidad electrónica» (2003/C 39/03).

I Plan Nacional de Accesibilidad 2004-2012, aprobado en Consejo de Ministros el día 25 de julio de 2003.

Congreso Internacional de personas con discapacidad. «No discriminación más acción positiva es igual a inclusión social». Madrid, 2003.

Real Decreto 1865/2004, de 6 de septiembre, por el que se regula el Consejo Nacional de la Discapacidad.

Ley Orgánica 1/2004, de 28 de diciembre, de Medidas de Protección Integral contra la Violencia de Género.

IV Plan Nacional de Acción para la Inclusión Social del Reino de España (2006-2008).

Plan de acción para las mujeres con discapacidad 2007, aprobado por el Consejo de Ministros de 1 de diciembre de 2006.

Convención Internacional sobre los Derechos de las Personas con Discapacidad. 13 de diciembre de 2006, Asamblea General de la ONU.

Ley 39/2006 de Promoción de la Autonomía Personal y Atención a personas en situación de dependencia, publicada en el BOE número 299 de 15 de diciembre de 2006.

Ley Orgánica 3/2007, de 22 de marzo, para la igualdad efectiva de mujeres y hombres.

\section{WEBs}

<http://www.catalogo-ceapat.org/> consultado el 30-04-2009.

$<$ http://sid.usal.es/colectivos.aspx> consultado el 30-04-2009. 
<http://usuarios.discapnet.es/disweb2000/index.htm>, consultado el 30-04-2009.

\section{ANEXO I}

Testimonio personal de la autora, con discapacidad visual ${ }^{37}$.

Desde agosto del año 2000, año en el que me sobrevino la discapacidad visual, y tras un proceso de incapacidad laboral temporal que culminó en septiembre de 2002 con la jubilación como pensionista, vengo realizando una continua actividad, gracias a las ayudas tecnológicas aportadas por la organización ONCE al facilitarme medios adecuados para paliar, en parte, esa importante deficiencia visual.

37. Baja visión por pérdida total de visión del ojo izquierdo y degeneración macular grave en el ojo derecho lo que me obliga a la utilización de ayudas técnicas para personas con baja visión. 